\title{
La Racionalidad como Ideal. Una refiexión desde la Teoría Elsteriana
}

\author{
The Rationality as Ideal. An Elsterian Approach \\ José Carlos Vázquez Parra ${ }^{1}$ \\ Recibido: 2/8/2016 / Aprobado: 7/3/2017
}

\begin{abstract}
Resumen
Dentro de la teoría filosófica, el habla de la irracionalidad de la acción no es algo nuevo, sin embargo, el proponer que la racionalidad es más un ideal que una norma puede llegar a sacudir algunos viejos paradigmas como el de la explicación de la acción racional. La propuesta Elsteriana de la racionalidad, viene a plantear una nueva perspectiva del calificativo de racionalidad y demuestra que gran parte de los comportamientos que se estiman como racionales realmente quedan fuera de dicha valoración. Esta nueva visión de la racionalidad de las acciones da cabida a cuestionarse sobre la posibilidad misma de actuar de manera racional, así como también del poder explicativo que algunas teorías de la racionalidad han sustentado en la presunción de esta.
\end{abstract}

Palabras clave: Acción, razón, comportamiento, deseos, creencias.

Abstract

From a philosophical theory perspective, a speech of irrationality of actions is not new. Though, rationality is an ideal not a rule. It challenges traditional paradigms of the explanatory power of rational action. Elsterian proposal of rationality seeks proposing a new vision of rationality of actions taking into account those occasions in which behaviors are considered to be rational, though they are not. This aspect of rationality of actions, enquiries the possibility of acting rationally, a doubt of the explanatory power that has sustained the rationality in the different theories of actions.

Key words: Action, reason, behavior, desires, beliefs.

\section{Introducción}

Hablar de racionalidad o en este caso, de irracionalidad, bien podría traer consigo diferentes formas de abordaje. Se puede profundizar en los planteamientos psicológicos en los que la irracionalidad tiende a relacionarse con el inconsciente freudiano o bien, a un abordaje desde la teoría económica, en la que la racionalidad a la par del interés es considerada como uno de los elementos característicos del llamado homo economicus. No obstante, sea cual sea la perspectiva que se decida, siempre se tenderá a hacer una relación entre este elemento valorativo (la racionalidad) y la teoría de la acción humana.

Dentro del ámbito de la filosofía, el hablar de la noción de irracionalidad no es algo nuevo, sin embargo, aunque lo irracional ya ha estado presente en el devenir histórico del pensamiento filosófico,

\footnotetext{
${ }^{1}$ Doctor en Estudios Humanísticos con Especialidad en Ética. Profesor, instructor e investigador en el Instituto Tecnólogico de Estudios Superiores de Monterrey, México. Campus Guadalajara, Escuela de Humanidades y Educación. Correo electrónico: jcvazquezp@itesm.mx
} 
será hasta los abordajes contemporáneos en que sea considerado como un tema de importante análisis. Según Rábade (1994: 30-35), lo irracional ha dejado de ser el calificativo negativo y caótico que la filosofía clásica consideraba y ha dejado atrás su sentido casi peyorativo para ostentar un lugar central en las discusiones filosóficas contemporáneas, donde dejando de ser "el desorden", toma el entendido de "un nuevo orden", es decir, como una nueva forma de explicar el comportamiento que se encuentra fuera de lo codificable por los estrechos cánones de la razón.

Es evidente que mientras las concepciones filosóficas del ser humano, por algunas corrientes del pensamiento, sigan siendo las de un ser primaria y básicamente racional, la explicación del comportamiento dejará muy poca cabida al estudio de lo irracional. Propuestas utilitaristas como las de Adam Smith o Jeremy Bentham, llegan a cuestionar de la posibilidad de un ente irracional, pues sus postulados de maximización obligan a concebir al hombre como un ser tanto interesado como intrínsecamente racional.Esto permite sugerir los siguientes cuestionamientos: ¿cuál es el lugar que puede ostentar la irracionalidad dentro de la teoría de la acción contemporánea?, ¿es factible dudar del lugar beneficioso en el que se ha colocado a la explicación racional?, ¿bajo qué situaciones se podría gestar esta duda?, de ser así, ¿cómo debería de reestructurarse el entendido de la teoría de la acción racional a partir de la posibilidad de irracionalidad?

Por lo anterior, es que es considerado de suma importancia el desarrollo de este artículo, ya que difícilmente podemos entender el papel que desempeña la irracionalidad en la teoría de la acción contemporánea, sin comprender primeramente el sentido de la irracionalidad misma, la forma en que esta altera algunas de las concepciones teóricas clásicas, sus implicaciones, así como la nueva valoración de la racionalidad que surgiría a partir de ello. De esta manera, el presente texto buscará responder a los anteriores cuestionamientos, precisando la noción misma de la irracionalidad, tanto desde su desarrollo histórico, como desde las algunas concepciones filosóficas contemporáneas. Para ello, se pondrá especial interés en los planteamientos propuestos por la teoría Elsteriana, la cual, no solo puede apreciarse como un planteamiento novedoso de explicación de la acción racional, sino también, como una reformulación de la presunción del ideal de racionalidad por medio de la posibilidad de la irracionalidad.

\section{Acercamiento histórico-filosófico de la noción de irracionalidad}

Cualquier análisis filosófico que pretenda una explicación histórica de un concepto, lleva, de manera casi directa, a un antecedente griego, sin ser la excepción, la noción de irracionalidad. Sin embargo, cabe señalar que la irracionalidad no encontraría un lugar pleno dentro de los análisis de los primeros filósofos helenos, ya que con una percepción aristotélica en la cual la razón ostenta un sitio casi inamovible dentro de la actuación humana, el abordaje de la irracionalidad no tiene cabida.

Para Aristóteles, la razón tiene un fundamento muy firme, ya que con base en los principios que plantea en sus obras y el carácter finalista de los actos, los procesos de racionalización tienen una seguridad muy afianzada, estimando que la racionalidad es el medio natural de comprender la acción tendiente a fines. Sin embargo, considerando las aportaciones Platónicas (Timeo: 52b) de que en la irracionalidad no hay conocimiento del ser, el estagirita propone una coextención entre el ser, la inteligibilidad y la determinación, planteando que ser y conocimiento están relacionados y se lleva a lo racional desde un nivel gnoseológico a otro prácticamente ontológico (Phys I, 4, 187 a 7). Esto es lo que nos lleva a considerar que la racionalidad aristotélica es apreciada prácticamente como una parte intrínseca del ser humano y que lo que cayera fuera de tales parámetros quedaba excluido a su vez de la razón como del ser (Rábade, 1994: 51). 
Esta concepción de la razón griega, ha llevado a que hoy sea muy complicado hablar de una noción de irracionalidad clásica, ya que con la adopción de los postulados griegos por parte de los pensadores de la Edad Media, el mundo greco-cristiano dejó poco espacio para el diálogo sobre la irracionalidad. Según Rábade (1994), con un orgullo racionalista y una fundamentación protegida por el método, la razón avanzó de manera segura durante mucho tiempo, ya que si a pesar del método existía alguna duda, aún se tenía la explicación de Dios, como ente del que se daba la derivación originaria de la razón. Sin embargo, entrando a la modernidad de la época ilustrada, los principios griegos fueron dejados a un lado, pues se buscaba, sin necesidad de negarlos, nuevos elementos explicativos de la acción. Este es el caso de la propuesta de David Hume, quien sustentando que gran parte de la acción humana requería del análisis de las emociones y las pasiones de esta, consideraba que la racionalidad requiere de nuevos fundamentos, entre los que sobresalen la intuición, la experiencia y la causalidad (Hume, 2005).

Hume hace mención en variadas ocasiones de la degradación manifiesta que se estaba dando en el desarrollo de la racionalidad, situación que llegaba a desacreditar el poder explicativo de la razón al tratar de analizar las acciones. Hume consideraba que la racionalidad había llegado a ser, no más que "un maravilloso e inteligible instinto de nuestras almas" (Hume, 2005: 1.3.16). En este análisis, Hume hace notorio que las propensiones, es decir, pasiones o deseos que tiene el individuo de hacer algo, deben estimarse como motivaciones inmóviles de los actos, lo cual se refleja en su famosa sentencia de que "la razón es, y solo debería ser, esclava de las pasiones" y que dichas pasiones son "verdaderas existencias originales" (Hume, 2005: 2.3.3.4).

Así, la reducción de la razón ante las propensiones y pasiones humanas, hace que se dé un giro en la interpretación de la racionalidad misma, destronándola del podio donde se ostentaba hasta ese momento. Hume propone que el sentido explicativo de las acciones, se generaba fundamentalmente a partir del análisis correlacional de los sucesos que afectaban al agente, considerando que es en el causalismo y no en la racionalidad, donde se alcanza el conocimiento y la explicación plena de la acción humana. Sin embargo, este pequeño avance en el análisis de la irracionalidad, se encontraría en un terreno árido con la llegada del pensamiento kantiano, el cual, respondiendo a las propuestas de Hume, consideraría que los planteamientos del filósofo escosés afectaban rotundamente a la explicación objetiva de los actos. Para Kant (trad. 2004) el estudio de la acción requiere de una noción que le dé estructura y objetividad, al considerar que el apegarse a ideas como las pasiones, las emociones o la experiencia, al encontrarse fuera de los límites del conocer, llevaban la acción a un terreno de subjetividad. Para este filósofo alemán, la racionalidad es la estructura que permite que el ser humano se acerque a un conocimiento objetivo de las cosas, por lo que todo aquello que queda fuera de la razón, simplemente no es posible llegar a conocerlo.

A pesar de este revés en el avance de una noción de irracionalidad, conforme se llegó a la época contemporánea, se fue gestando una nueva desconfianza a la explicación plenamente racional y se desarrollaron incluso algunos puntos de enemistad con esta. Planteamientos como los de Hegel, Schopenhauer y Nietzsche abrieron lugar para el estudio filosófico del irracionalismo y colocaron a la razón, ya no como un parámetro que decidía sobre los actos, sino como una justificación y manera de explicar los elementos que constituían las acciones. De esta manera, el hombre se encontraría frente a una gran variedad de nociones que requieren descifrarse y comprenderse para llegar a entender la actuación humana y lo irracional como aquello que se consideraba quedaba fuera de la norma y sobre lo que el deseo humano luchaba por racionalizar.

Sin embargo, dentro de la teoría de la acción contemporánea, la lucha por la posibilidad de una noción de irracionalidad aún genera 
profundas discusiones teóricas, entre aquellos filósofos que se siguen apegando a la presunción de racionalidad y aquellos que estiman que esta es un ideal que aleja al agente de la realidad en la que se desenvuelve. Propuestas como las de Amartya Sen o Jonathan Glover vienen a exponer que el individuo no puede simplemente abandonarse a una racionalidad totalizadora e ideal, pues ello demerita cuantiosamente a la acción misma, al no considerar ni el entorno en el que se genera, ni los elementos que la constituyen. Así, el presente artículo puede adentrarse en el análisis de la racionalidad, pero ahora desde la teoría de la acción.

\section{La posibilidad de Irracionalidad en la teoría de la Acción}

Un planteamiento muy clarificador sobre la disyuntiva entre la presunción de un ideal de racionalidad o la posibilidad de una noción de irracionalidad, es el que se puede rescatar de Amartya Sen en su obra La idea de la Justicia (2010). Este filósofo bengalí considera que la presunción de razón, a la que se aferran muchos teóricos de la acción (fundamentalmente aquellos que se respaldan en la teoría de la elección racional), se apega notoriamente a la perspectiva de esquemas ideales del institucionalismo trascendental, que se caracteriza por identificar aquellos esquemas ideales de ciertas nociones que servían de sustento para propuestas teóricas, como es el caso de la justicia, la libertad, la autonomía o la razón, sin ocuparse verdaderamente de las sociedades reales (Álvarez, 2010).

Según Sen (2010: 38), dentro de este institucionalismo trascendental podemos encontrar los planteamientos de Locke, Rousseau, Kant, Rawls y Nozick, quienes consideraban que conceptos como la justicia, la razón, la libertad, entre otros, debían basarse en esquemas ideales, aplicados en un conjunto de instituciones determinadas en las que se plasmen esquemas sociales perfectamente estructurados, de tal suerte que estos incluyeran tanto al comportamiento correcto como a las instituciones correctas. En contraste con esta perspectiva basada en ideales, está aquella que se sustenta en los resultados observados (o realizaciones), es decir, en comportamientos reales del agente, en lugar de presuponer la observancia general de un comportamiento ideal. Dentro de los teóricos que pertenecieron a este grupo se encuentra fundamentalmente Karl Marx quien en gran medida basó sus propuestas teóricas en un análisis social comparado y no así con estructuras sociales ideales (Sen, 2010: 39).

Sen valora que este último enfoque de resultados observados, se conecta mucho más con las necesidades y aspiraciones de los individuos, pues se sustenta en aspectos concretos de las instituciones y no así, en la racionalidad abstracta de las normas, como lo propone el institucionalismo trascendental. Por ello, este filósofo y economista estima que aquellas propuestas teóricas, que se sustentan en un ideal de razón, tienen claras lagunas y limitaciones al relacionarse con la realidad y el entorno del agente, en contraste con aquellas teorías que se basan en el análisis de los éxitos y fracasos de sociedades específicas y que dan pauta a una racionalidad limitada.

Esta división señalada por Sen, entre una teoría de la acción basada en una racionalidad ideal y otra que se sustenta en la necesidad de análisis de la realidad del agente, ha generado nuevo punto de reflexión entre los teóricos contemporáneos, pues mientras algunos continúan asiduos a la presunción ideal de la racionalidad como un aliado fundamental en la tarea de mejorar las sociedades y maximizar las acciones, para otros, como Jonathan Glover (2001), esta confianza absoluta en la razón puede ser sumamente arriesgada al momento de actuar, al existir la posibilidad de dar cabida a ideologías basadas en creencias ciegas o impuestas, es decir, sustentadas en una mala racionalidad. 
Al igual que Sen y Glover, teóricos como Calvert (2000: 568), Jerkins (1996: 7), Hardin (1995), McInnes (2004: 533) y Anscombe (1951: 50), cuestionan la presunción de la razón, pues de manera contraria a los planteamientos de un ideal de razón, estos teóricos apuestan a la presencia de elementos independientes que se separan del carácter maximizador y que intervienen en un proceso de racionalización de la actuación del agente y no simplemente en una racionalidad dada.

Randall Calvert (2000: 568) y Jerkins (1996: 7) han considerado que aunque la racionalidad ideal es atractiva en la búsqueda de explicar el comportamiento humano, existen otras opciones. Por ejemplo, Calvert (2000: 568) expone que propuestas como la teoría de la elección racional, que presumen una racionalidad en los agentes, no dan espacio al estudio de la identidad del individuo, por lo que estima que una metodología identitariaexpresiva es una mejor opción para explicar el comportamiento, pues en esta, la identidad propia del agente (raza, género, edad, nacionalidad entre otras manifestaciones de la identidad) se funde en la toma de sus decisiones individuales.

Hardin (1995: 7) y McInnes (2004: 533), consideran, contrarios a Calvert, que esta relación causal entre identidad y acción es oscura y en la mayoría de las ocasiones, más que ser evidente es algo a lo que se le atribuye un poder explicativo de que carece. Sin embargo, al tratar de explicar una acción, lo que en realidad se cuestiona es por las razones que el agente tuvo para realizarla, dando pauta a una necesidad de explicar el causalismo interno del actuar, a partir de sus razones. Ahora bien, un tercer punto de vista se encuentra en Anscombe (1991: 50), quien considera, complementando a Hardin, que no siempre se tiene acceso directo a las motivaciones internas de las personas, es decir a sus razones (deseos y creencias), por lo que el análisis causal, en la mayoría de las veces, es el único medio que se tiene para alcanzar un real entendimiento de las acciones de los agentes, más allá de sus razones. De esta manera, la teoría de la acción se ha desarrollado paralelamente al conflicto entre aquellos teóricos que postulan a la racionalidad como una norma de ideales de comportamiento que deben ser cubiertos con el fin de maximizar y dar certeza a las consecuencias de las acciones, contra aquellos que consideran que más que una elección utópicamente racional, el agente requiere acercarse lo más posible a generar escalas de preferencias, y con ello elecciones tanto reales como verdaderas, siendo la racionalidad y sus elementos un proceso para alcanzar dicho objetivo.

La irracionalidad dentro de la teoría de la acción puede ubicarse como una situación residual que surge a partir de la no consecución del calificativo de racionalidad que ya no debe ser considerado como una presunción ideal de toda acción, sino simplemente, como un proceso evaluativo de la actuación del agente. Propuestas como la de Donald Davidson o la de Jon Elster vendrían a poner especial atención a el desarrollo de dicho proceso de racionalidad de la acción, valorando que el decir que las acciones son racionales debe traer consigo un nivel de escrutinio que no es fácilmente alcanzable, lo que puede ocasionar que a pesar de la intención del agente, este termine actuando irracionalmente.

\section{La posibilidad de Irracionalidad desde la propuesta teórica de Donald Davidson}

En 1980, en que sale a la luz su obra Acciones, Razones y Causas, Donald Davidson (trad. 1995: 17-36) propone lo que se convertiría en la postura tradicional y característica del resto de sus escritos, es decir, su entendido de la racionalidad como la explicación de la acción mediante razones (deseos y creencias). Davidson considera que el poder dar razón de los actos, conlleva un nivel intrínseco de racionalidad, ya que simplemente, la facultad de dar razones denota cierta intencionalidad de parte del agente, elemento que hace la diferencia entre las acciones que se realizan y los sucesos que simplemente pasan (Caorsi, 2008: 653). 
Dicha presunción intrínseca de intencionalidad y con ello de racionalidad, aunque lleva a que Davidson (trad. 1995) considere que la irracionalidad no es algo que pueda darse por sí misma, no lo cierra a la posibilidad de la presencia de esta, pues valora que dicha irracionalidad puede configurarse aun habiendo cierto grado de racionalidad. Para este filósofo norteamericano, existe una distinción entre la irracionalidad y la no racionalidad y explica que la primera solo se configura a partir de la inconsistencia interna de la acción, mientras que la segunda, se da por la imposibilidad de dar razones de los comportamientos, situación que solo puede gestarse dentro de los sucesos.

Davidson considera que mientras los sucesos pueden no ser racionales, las acciones solo pueden llegar a ser irracionales, pues esto deriva no de su falta de racionalidad, sino de la inconsistencia o contradicción en los procesos internos de la acción. De esta forma, Davidson plantea que un sujeto actúa de manera irracional solo cuando lo hace en forma incoherente con la estructura consistente de sus creencias, actitudes, emociones, intenciones o deseos, estimando que una acción no racional es imposible.

Para Davidson (1995), la irracionalidad se configura como un error en el proceso de la generación de las razones que respaldan la acción, lo que aunque no desecha la racionalidad intrínseca de la actuación, si afecta claramente la presunción de una noción ideal, al considerarla inconsistente y por ende limitada. Aunque algunos contemporáneos de su época, consideraron la teoría de Davidson como una propuesta bastante atractiva, otros, como es el caso del noruego Jon Elster, cuestionaron que el parámetro de la consistencia fuera suficiente para calificar la racionalidad de la acción. Para Elster (2007: 23), la posibilidad de un calificativo de racionalidad de la acción requiere de un análisis exhaustivo previo que lleve consigo un escrutinio crítico de los elementos que constituyen la actuación del agente, por lo que estima que tanto la presunción interseca de racionalidad Davidsoniana, como su noción de irracionalidad sustentada en inconsistencia resulta muy limitada. De esta manera, es importante analizar el planteamiento Elsteriano y como su proceso de calificación de la racionalidad de la acción, puede llevar a gestar una perspectiva nueva de la irracionalidad.

\section{La concepción Elsteriana de la Irracionalidad}

Para comprender de la irracionalidad elsteriana, es importante hacer el abordaje desde su noción de racionalidad, ya que como se mencionó anteriormente, la concepción de irracionalidad para algunos teóricos de la acción como lo es en el caso del profesor Elster es percibida desde un sentido residual.

Elster (1999: 13) propone que toda acción que se considere racional debe implicar tres operaciones de optimización: hallar la mejor acción para las creencias y los deseos dados, formar la creencia lo mejor fundada para una prueba dada y acumular la cantidad óptima de pruebas para los deseos dados y las creencias previas. Estas operaciones, conllevan de manera intrínseca la necesidad de valorar individualmente los elementos que constituyen la acción, no solo desde su calificativo de consistencia, como lo plantea Davidson, sino también desde su valoración de racionalidad, esto con el fin de alcanzar un nivel descriptivo y explicativo óptimo de la acción.

Con base en lo anterior, para llegar a un análisis pleno de la racionalidad del acto, y por ende de la irracionalidad elsteriana, es que se debe considerar el papel que desempeña cada uno de los elementos de la acción, es decir, la manera en que se generan los deseos y la forma en que interactúan con las creencias, así como la intención de estructurar una escala de preferencias en la que pueda ejercerse la elección. Para la racionalidad elsteriana, no es suficiente el análisis racional de la acción en general, 
ya que el calificativo racional implica a su vez la racionalidad individual del resto de los elementos de esta. Este sentido, la irracionalidad desde el enfoque elsteriano, llega a ser comprendida a partir de un abordaje a los limitantes que se presentan en la interacción de la acción con sus elementos, por lo que para poder llegar a una propuesta, conviene hacer un análisis por separado de cada una de las partes de la acción.

Como primer elemento de la acción, los deseos deben cubrir dos parámetros con la pretensión de ser valorados como racionales. Primeramente, los deseos deberán ser consistentes, lo cual implica que tienen existir dentro del mundo de lo posible, tomando en cuenta todas las restricciones físicas, económicas, legales y psicológicas que enfrenta el individuo, debiendo pertenecer al conjunto de oportunidad del agente (Elster, 1989:28). Por otro lado, los deseos deben demostrar que son autónomos, es decir, que estos han sido formados correctamente y no mediante mecanismos causales desdeñables, que operando a espaldas del agente, lo hagan sentir avergonzado de tener consciencia de ellos (Elster, 1989: 49).

Para Elster (1999: 15), no se puede ser racional si se es juguete de procesos psíquicos que, sin que lo sepa el agente, moldean sus deseos, por lo que tener en consideración la autonomía de lo que se desea y la manera en que se hace se vuelve un punto fundamental para demostrar la racionalidad de estos. Situaciones como las motivaciones inconscientes, las adicciones, los precompromisos, las preferencias contra-adaptativas, así como el seguimiento de normas sociales o morales, pueden cuestionar la autonomía de los deseos y hacer que estos queden fuera de los límites de la racionalidad y afecten con ello la valoración de la acción en general.

Con respecto al segundo elemento de la acción, al igual que se da en los deseos, para Elster la racionalidad de las creencias debe juzgarse conforme un doble sistema de valoración; es decir, la consideración primera de su consistencia para posteriormente, calificar su racionalidad en sí.
En cuanto al primer nivel de evaluación, Elster (1988: 14) señala como consistentes a aquellas creencias que se encuentran en conformidad con las leyes de la probabilidad, es decir, que son posibles y realísticamente creíbles, cuando existe un mundo de posibilidades en el cual sean verdaderas, creídas y donde, sobre todo, no sea posible que se derive una contradicción a partir de ellas. El segundo nivel de valoración, el filósofo noruego plantea que la racionalidad de las creencias se alcanzará a partir de la relación que tienen estas con la evidencia que se posee en concordancia con tal información, con la historia causal de esta y poniendo una especial atención en la recabación óptima del material que sustente la evidencia a partir del juicio. Sin embargo, la formación de las creencias puede fallar, lo cual desde la perspectiva Elsteriana puede darse a partir de diferentes contextos generadores de irracionalidad, los cuales pueden ir desde la simple ignorancia de datos básicos de la creencia (Elster, 1999: 31), hasta un elaborado proceso de autoengaño que se genera por el agente para reducir la disonancia cognitiva de sus acciones (Elster, 1999: 28). Sin embargo, sea cual sea, se habla de una situación que afecta tanto a la creencia, como a la posterior estructuración de las preferencias, por lo que ambos elementos se ven inevitablemente relacionados en este punto.

Es importante hacer hincapié en que muchos de los factores que pueden afectar la racionalidad de los deseos igual pueden presentarse al alterar la valoración de las creencias o bien a la estructuración de las preferencias, por lo que bajo esta perspectiva, la racionalidad es llevada a un nivel mucho más profundo, el cual puede calificarse tanto en lo general como en lo particular de cada una de sus partes, y considerarse de manera aislada, así como en su interacción con el resto de los elementos de la acción.

Con base en lo anterior, se puede apreciar que conforme a esta propuesta, los nuevos requerimientos fijados por Elster para lograr el calificativo de racionalidad, hicieron que la 
evaluación de la acción se volviera cada vez más minuciosa y ocasionara una racionalidad difícil de ser alcanzada. Esto dio pauta a que un sinnúmero de acciones que anteriormente eran estimadas como racionales, bajo estos nuevos parámetros, no logren cumplir todos las exigencias elsterianas y dieran cabida a un estado mayoritariamente irracional, donde, como diría el noruego, la teoría de la racionalidad se encuentra claramente limitada.

La propuesta elsteriana de la racionalidad de la acción, puede ser concebida como un planteamiento altamente restrictivo (Casas, 2001) que al buscar generar un proceso de escrutinio crítico del calificativo de racionalidad, llevó a que el agente se viera ante la difícil realidad de que sus acciones pocas ocasiones llegaban a ser óptimamente racionales. Sin embargo, la irracionalidad, vista como una noción mucho más cotidiana dentro de la teoría de la acción elsteriana no debe ser considerada como una situación que amerite el que se abandone el privilegio explicativo de la racionalidad, pues no se puede negar que aun sabiéndose irracional, el agente siempre busque ser lo más racional posible. La racionalidad, debe valorarse como un parámetro que permita acercar al agente a alcanzar una mayor certeza y optimalidad en la comprensión de sus acciones y sus consecuencias y no simplemente como aquel calificativo ideal que alejaba a las acciones de la realidad misma.

Aunque conforme a la teoría Elsteriana de la racionalidad, el agente difícilmente alcanzará un calificativo racional óptimo de sus acciones, esto no demerita que el proceso en sí permita que el individuo alcance un mayor conocimiento de su comportamiento, así como una consciencia más plena de los elementos que conforman sus acciones. Lo anterior es lo que lleva a que como estudiosos de la acción, se deba elegir cualquiera de las dos posibilidades para proseguir con el análisis de la actuación humana. Por un lado se puede aceptar algunas de las alternativas teóricas que buscan subsanar o evitar una valoración estricta con la pretensión de aferrarse a una presunción de racionalidad como método para explicar el comportamiento humano. O bien, se puede adoptar la propuesta elsteriana, reconociendo que el valor real de la racionalidad no se encuentra necesariamente en su poder explicativo o en la posibilidad de ser alcanzada por el agente, sino más bien, en la profunda y estricta reflexión que se logra por medio del proceso de valoración de la racionalidad.

De esta manera, más que el calificativo de racionalidad, es el deseo de actuar racionalmente, el que con su poder motivador logra que el agente alcance un proceso reflexivo más detallado y minucioso, subsanando la mayor cantidad de limitaciones de la racionalidad, así como alcanzar una mayor certidumbre sobre las consecuencias de sus actos.

La teoría de la racionalidad Elsteriana, más que contradecir a los planteamientos que la anteceden, viene a complementarlos por medio de una valoración más estricta del calificativo de razón, que da cabida a la posibilidad de cuestionar a todos aquellos comportamientos que no respondían a las necesidades mínimas de optimalidad y que anteriormente llegaban a considerarse como racionales.

El presente artículo ha buscado reflexionar sobre esta intención elsteriana de restablecer la valoración de la racionalidad a partir de un nuevo enfoque, el cual, no solo exige la modificación de la noción tradicional de razón, sino también obliga a acercarse a la irracionalidad y a considerar el papel que esta desempeña dentro de la explicación contemporánea de la teoría de la acción humana.

\section{Consideraciones finales}

Algunas de las preguntas que se propusieron al inicio de este artículo, hacían parecer que este tendría una amplitud demasiado ambiciosa; sin embargo, una vez llegado a este punto, podemos estimar con la explicación brindada, que se logra una buena aproximación para responder a los 
cuestionamientos planteados: ¿cuál es el lugar que debe ostentar la irracionalidad dentro de la teoría de la acción contemporánea?, ¿es factible dudar del lugar beneficioso en el que se ha colocado a la explicación racional?, ¿bajo qué situaciones se podría gestar esta duda?, ¿de ser así, cómo debería de reestructurarse el entendido de la teoría de la acción racional a partir de la posibilidad de irracionalidad?

La posibilidad que se abre con la teoría elsteriana de la racionalidad, al mostrar la generalidad con la que la irracionalidad se presenta dentro del comportamiento humano, permite contestar algunas de las preguntas planteadas, ya que el entendido de que la mayoría de las acciones humanas se encuentran limitadas racionalmente, permite tanto cuestionar el poder explicativo de la racionalidad, como comprender el papel tan importante que desempeña la compresión de la noción de irracionalidad en la filosofía y en la teoría de la acción contemporánea. Sin embargo, así como se presentó esta generalización irracional no debe tomarse en cuenta desde un sentido anulador del poder explicativo de la racionalidad, sino más bien, como una posibilidad de reestructurar el poder descriptivo de esta, a partir de su valoración motivacional y la tendencia natural que tienen los agentes por alcanzar el ideal de razón.

Así, la teoría de la acción racional, planteada desde la perspectiva elsteriana, obliga a que como estudiosos de la elección, decisión y acción humana, se deba llevar el análisis de la acción y sus elementos a los límites de la posibilidad racional, la cual aunque no consigue el calificativo de una racionalidad ideal, permite un análisis crítico bastante exhaustivo, con el que se puede tener una mayor certidumbre sobre la explicación de la acción y sus consecuencias, ya sea a partir de los parámetros fijados por la racionalidad o desde sentido residual de la irracionalidad.

\section{Referencias bibliográficas}

Rábade, S. (1994). La razón y lo irracional. Madrid: Complutense.

Elster, J. (1999). Juicios Salomónicos. Barcelona: Gedisa.

Davidson, D. (1995). Ensayos sobre acciones y sucesos. Barcelona: Crítica.

Caorsi, C. (2008). La filosofía de la acción de Donald Davidson. In G. Leyva, Filosofía de la acción (pp. 653-684). España: Sintesis.

Elster, J. (2007). La explicación del comportamiento social: mas tuercas y tornillos para las ciencias sociales. Barcelona: Gedisa.

Elster, J. (1989). Tuercas y Tornillos. Barcelona: Gedisa.

Elster, J. (1988). Uvas Amargas. Sobre la subversión de la racionalidad. Barcelona: Peninsula.

Elster, J. (1995). Estudios sobre la racionalidad. Lectura pronunciada en la ceremonia en que se le condecía el título de Doctor Honoris Causa por la Universidad de Valencia. Valencia.

Simon, H. (1982). Models of Bounded Rationality. Cambridge: MIT Press.

Lazarsfeld, P. F. (1962). The language of social research: a reader in the methodology of social research. Chicago: Free Press.

Hume, D. (2005). Tratado de la Naturaleza Humana. México: Porrúa.

Kant, I. (2004). Critica de la Razon Practica. México: Porrúa.

Casas, J. (2001). Estudio Introductorio. In J. Elster, Las limitaciones del paradigma de la elección racional. Las ciencias sociales en la encrucijada. (pp. 11-42). Valencia: Institución Alfons el Magnánim. 
Episcopado, d. M. (2012). Pan de la Palabra. Tlaquepaque, Jalisco, México: San Pablo.

Álvarez, F. (2010). "La propuesta inmanentista de Amartya Sen para la justicia global”. Isegoria (43), 617-630.

Sen, A. (2010). La idea de la justicia. México: Taurus.

Glover, J. (2001). Humanidad e Inhumanidad. Madrid: Catedra.

Calvert, R. (2000). Identity, expresion, and rationalchoice theory. In I. K. Milner, Political Science: The State of the Discipline (pp. 568596). Nueva York: Columbia University Press.

Jerkins, R. (1996). Social Identity. Londres: Routledge.

Hardin, R. (1995). One for All. Princeton: Princeton University Press.

McInnes, J. (2004). The sociology of identity: social science or social comment? British Journal of Sociology, , 55 (4), 531-543.

Anscombre, E. (1991). Intención. (A. I. Stellino, Trans.) Barcelona: Universidad Autónoma de México.

Sen, A. (1976). Los tontos racionales. Una critica de los fundamentos conductistas de la teoria económica. Philosophy and Public Affairs (6), 315-344.

Sen, A. (2010). La idea de la justicia. México: Taurus.

Simon, H. (1982). Models of Bounded Rationality. Cambridge: MIT Press. 